\title{
Serum RANKL levels in Chinese patients with ankylosing spondylitis: a meta-analysis
}

\author{
Feifei $\mathrm{Ni}^{1 \dagger}$, Yanchao Zhang ${ }^{2 \dagger}, \mathrm{Yi} \mathrm{Peng}^{3}$, Xiaoxiao Peng ${ }^{4}$ and Jianjun $\mathrm{Li}^{1^{*}}$
}

\begin{abstract}
Objective: We aimed to determine the association between serum receptor activator of nuclear factor-kappa $B$ ligand (sRANKL) levels and ankylosing spondylitis (AS) in Chinese patients.

Methods: The PubMed, Cochrane Library, Embase, Chinese Biomedical Database, Web of Science, China National Knowledge Infrastructure, VIP, and Wan Fang databases were searched for studies conducted before October 1, 2020, without language restrictions. STATA version 12.0 and Revman version 5.3 were used to analyze the data. The standard mean differences (SMDs) and corresponding 95\% confidence intervals (95\% Cls) were calculated.

Results: Twelve clinical case-control studies, including 585 patients with AS and 423 healthy controls, were included. The combined SMD for SRANKL suggested that the sRANKL level was significantly higher in Chinese patients with AS than in healthy controls (SMD: 3.27, 95\% Cl 2.11-4.43, $P<0.00001)$. Serum RANKL-related factor osteoprotegerin (OPG) levels (SMD: $0.86,95 \% \mathrm{Cl} 0.09-1.64, P<0.03$ ) were lower in the Chinese patients with AS than in healthy controls, and the RANKL/OPG ratio (SMD $=1.05,95 \% \mathrm{Cl} 0.64-1.46, P<0.00001)$ in Chinese patients with AS was approximately the same as that of healthy controls. Subgroup analysis indicated that patients from North and South China had higher sRANKL levels than controls; the sRANKL levels of patients from South China were higher in the subgroup with a Bath Ankylosing Spondylitis Functional Index (BASFI) of $>4$ than those of patients in other subgroups. In terms of duration, patients with AS for $>8$ years had higher sRANKL levels than health controls. Other subgroup analyses were conducted by region, language, source of control, age, and Bath Ankylosing Spondylitis Disease Activity Index (BASDAI). In these subgroups, the sRANKL levels were significantly higher in the patients with AS than in healthy controls. The BASFI and BASDAI were sources of heterogeneity.
\end{abstract}

Conclusions: The SRANKL levels are higher in Chinese patients with AS, especially among those from South China. sRANKL levels may be positively correlated with the pathogenesis of AS among Chinese patients.

Keywords: RANKL, Ankylosing spondylitis, Pathogenesis, Meta-analysis, China

*Correspondence: Chinan888999@163.com

${ }^{\dagger}$ Feifei Ni and Yanchao Zhang have equally contributed as co-first authors

${ }^{1}$ Department of Orthopaedics, Shengjing Hospital of China Medical

University, Sanhao Street No. 36, Heping District, Shenyang, Liaoning 110004, People's Republic of China

Full list of author information is available at the end of the article

\section{Introduction}

Ankylosing spondylitis (AS) is a type of inflammatory arthritis, which belongs to the spondyloarthritis family that includes reactive arthritis and psoriatic arthritis [1]. There are a large number of patients with AS worldwide. The prevalence of AS in China is approximately $0.3 \%$, which is approximately 4 million people of China's population of 1.4 billion people [2]. Hence, AS leads to serious economic burdens on families and the society [3]. Meanwhile, an in-depth understanding of the pathogenesis of AS may address the problems associated with delayed original author(s) and the source, provide a link to the Creative Commons licence, and indicate if changes were made. The images or other third party material in this article are included in the article's Creative Commons licence, unless indicated otherwise in a credit line to the material. If material is not included in the article's Creative Commons licence and your intended use is not permitted by statutory regulation or exceeds the permitted use, you will need to obtain permission directly from the copyright holder. To view a copy of this licence, visit http://creativecommons.org/licenses/by/4.0/. The Creative Commons Public Domain Dedication waiver (http://creativeco mmons.org/publicdomain/zero/1.0/) applies to the data made available in this article, unless otherwise stated in a credit line to the data. 
diagnosis of AS and insufficient therapeutic strategy for the disease [4]. Features of AS include the anatomical distribution of the affected joints, types of joint damage, extra-articular manifestations, and sex-related distribution and eyes, intestine, and skin effects $[5,6]$. Recent studies have suggested that cytokines, including leptin, adiponectin, and resistin, may play important roles in the pathogenesis of AS [7]. When inflammation occurs, new bone formation leads to bone sclerosis, which can lead to AS; reports have indicated that osteopenia and osteoporosis both occur in AS [8]. Receptor activator of NF-kappa B ligand (RANKL), which was first found on the surface of osteoblasts, also plays an important role in different stages of bone cell metabolism $[9,10]$. It is a transmembrane protein that belongs to the tumor necrosis factor (TNF) superfamily [11], which comprises 316 amino acids [12], and is mainly expressed in the bone surface and lymphoid tissue [13]. RANKL and its RANKL receptor play important roles in bone metabolism and the immune system [14]. RANKL adherence to the bone surface is necessary to promote osteoclast differentiation, activation, and survival and accelerates the progress of osteoclast biology $[15,16]$. However, osteoclast overactivation leads to bone resorption and has been observed in a variety of bone diseases, such as bone metastasis and osteoporosis; likewise, RANKL is necessary for osteoclast differentiation and immune regulation [17]. Magnetic resonance imaging (MRI) results have indicated that bone inflammation and osteitis are associated with the presence of RANKL [18]. Targeted deletion of RANKL in bone cells prevents osteoclast formation [19].

Recently, several studies have shown that serum RANKL (sRANKL) levels are correlated with AS disease activity and are significantly elevated in patients with AS $[20,21]$. However, other studies have found no clear link between RANKL and AS in Asians [22-24]. The relationship between sRANKL and AS among Chinese patients is still unclear. Thus, we performed this meta-analysis to assess the link between sRANKL level and AS in Chinese individuals and to determine which diagnosis and treatment of AS are more convenient and effective.

\section{Materials and methods}

\section{Literature search}

We searched the following electronic databases without any language restrictions: PubMed, Cochrane library, Embase, Chinese Biomedical Database (Chinese database), Web of Science, Chinese National Knowledge Infrastructure (Chinese database), VIP (Chinese database), and Wan Fang (Chinese database). The search strategy was highly sensitive and was performed using a combination of the following keywords and $\mathrm{MeSH}$ terms:
"Ankylosing Spondylitis" or "Ankylosing Spondylarthritides" or "AS" and "RANKL" or "OPGL Protein" or "Osteoclast Differentiation Factor" or "Osteoprotegerin Ligand" or "TRANCE Protein."

\section{Selection criteria}

The selection criteria were as follows: (1) only casecontrol studies in the population to explore the relationship between sRANKL and AS; (2) patients who meet the modified New York criteria or Assessment of SpondyloArthritis international Society [25, 26]; (3) articles should be associated with sRANKL concentration; (4) sufficient and original data; and (5) articles in Chinese should have an English abstract. Studies that did not meet the selection criteria were excluded. If one author published different studies about the same topic, the most recently published or the study with the largest sample size was selected. All studies identified were investigated independently for eligibility by two of the authors (Feifei Ni and Yanchao Zhang), who browsed the title and abstract to select eligible studies. If any reviewer browsed a title or an abstract that met the screening criteria, the full text was browsed.

\section{Data extraction}

From the selected articles, two researchers (Feifei Ni and Xiaoxiao Peng) independently extracted and recorded the required information. Disagreements over data or included studies were resolved through discussion of all items. The recorded information included surname of initial authors, region, language, publication years, age, duration, the Bath Ankylosing Spondylitis Functional Index (BASFI, used to define and to monitor physical functioning in patients with ankylosing spondylitis), the Bath Ankylosing Spondylitis Disease Activity Index (BASDAI, used to measure patient-reported disease activity in patients with ankylosing spondylitis) [27], sRANKL detection method, sRANKL and osteoprotegerin (OPG) levels in the cases and controls.

\section{Quality of the study}

Two researchers (Feifei Ni and Xiaoxiao Peng) used the Newcastle-Ottawa Scale (NOS) to assess the quality of the included studies [28]. The NOS comprises three aspects: (1) subject selection: 0-4; (2) comparability of subject: $0-2$; and (3) clinical outcome: $0-3$. The NOS scores range from 0 to 9 with two levels of included studies: low quality (0-6) and high quality (7-9). When the two researchers disagreed or when there were discrepancies in the NOS score of a study, a third reviewer intervened. 


\section{Statistical analyses}

The relationship between sRANKL levels and AS susceptibility was assessed using the standardized mean differences (SMDs) and 95\% confidence intervals (95\% CIs). Cochran's Q-statistic $(P<0.05$ was considered significant) and $I^{2}$ tests were used to quantify heterogeneity among studies [29]. The random effects model was used when heterogeneity was significant $\left(P<0.05\right.$ for the Q test or $I^{2}$ test exhibited $\left.>50 \%\right)$; otherwise, the fixed-effects models were used [30]. When heterogeneity was significant, subgroup analysis was performed to find the potential reasons for the difference in sRANKL levels between patients with AS and healthy controls. In addition, sensitivity analysis was used to assess if a single study had an impact on the whole assessment. The impact of publication bias was analyzed using Egger's test $(P<0.05$ was considered significant), which can be used to evaluate the funnel plot asymmetry that reveals potential publication bias [31] [32]. The data were analyzed using the software Review Manager 5.3 and STATA version 12.0.

\section{Results}

Inclusion criteria

We selected 499 potentially relevant articles from eight databases. After deleting duplicates, 348 records remained. By skimming the titles and abstracts, we excluded 258 papers due to at least one of following reasons: (1) 6 articles were comments, 5 were letters, 25 were reviews, and 9 were editorials; (2) 156 were not related to the research topics; and (3) 57 were not on human studies. Full-text articles from the remaining 90 articles were reviewed again and 60 trials were excluded (30 were not case-control studies, 18 were not relevant to RANKL, and 12 were not relevant to AS), leaving 30 studies to the next selection step. After studies that were not related to the Chinese population and those lacking data integrity were removed, 12 studies were finally selected [33-44] (Fig. 1).

\section{Features of the studies}

Twelve studies, comprising 585 patients with AS and 423 controls, were included in accordance with the selection criteria. The basic features of the studies are shown in Table 1 . sRANKL levels in all 12 studies were tested using enzyme-linked immunosorbent assay. The

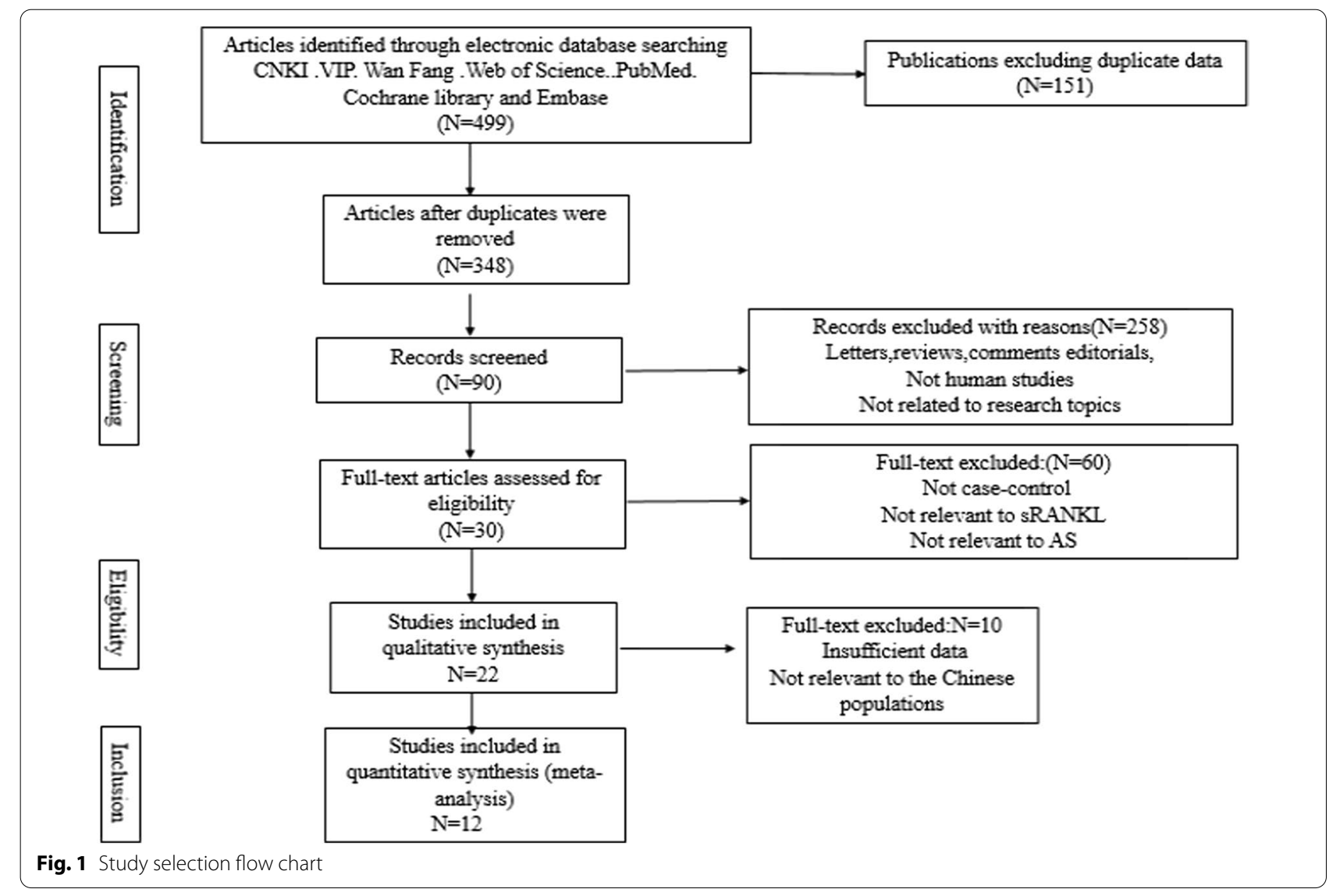




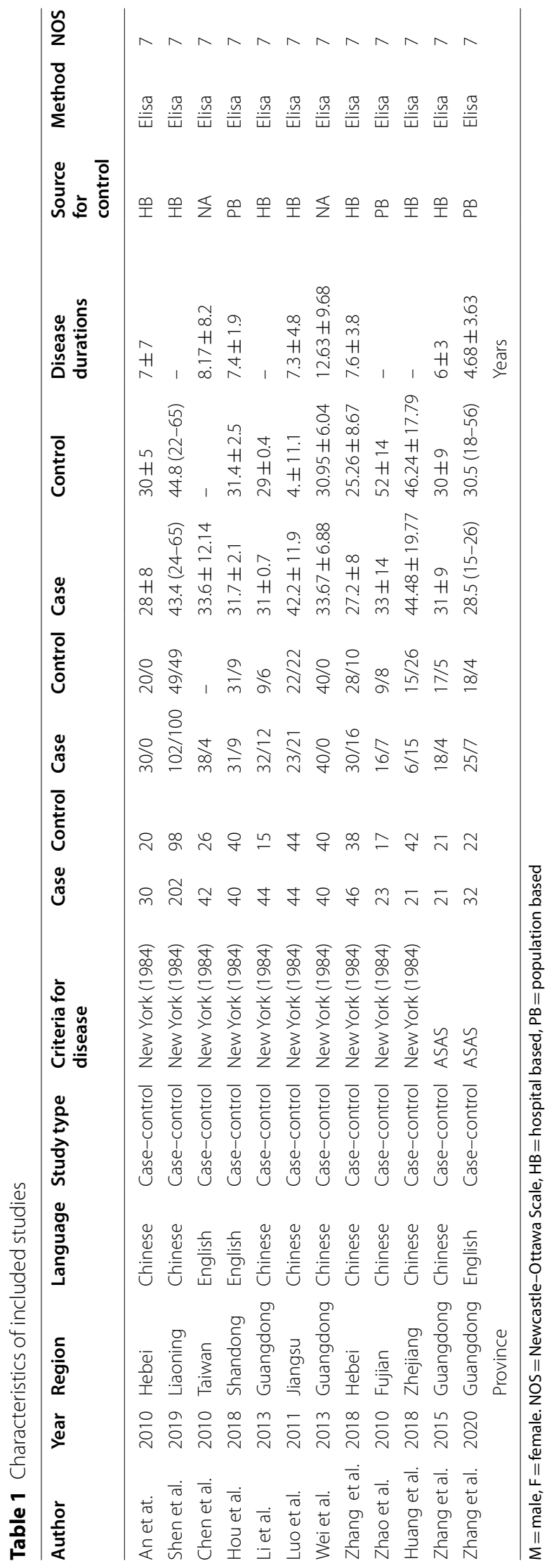


methodological quality assessment using the NOS is shown in Table 1.

\section{Meta-analysis in AS}

Significant heterogeneity was found in the 12 studies $\left(P<0.00001, I^{2}=97 \%\right)$, and the random-effects model showed that the sRANKL levels in patients with AS were statistically different compared with those in controls $(\mathrm{SMD}=3.27,95 \%$ CI 2.11-4.43, $P<0.00001$ ) (Fig. 2). Subgroups, including language, source of control, age, BASFI and the BASDI, were analyzed. In terms of these subgroups, the sRANKL levels of patients with AS were significantly higher than those of controls (Figs. 3, 4).

Subgroup analysis by region was divided into patients from North and South China because of the geographical differences in China's population distribution. Patients with AS in the two groups obviously had higher sRANKL levels than controls, although the patients from the south had higher sRANKL levels $(\mathrm{SMD}=3.55$, 95\% CI 2.18-4.92, $P<0.00001)$ than those of patients from the North $(\mathrm{SMD}=2.62,95 \%$ CI $0.34-4.9, P<0.02)$. Further subgroup analysis indicated that a BASFI of $>4 \quad(\mathrm{SMD}=3.14,95 \% \mathrm{CI} 2.69-3.58, P<0.00001)$ and duration of $\leq 8$ years $(\mathrm{SMD}=2.02,95 \%$ CI $1.03-3.02$, $P<0.0001)$ had a positive correlation in patients with AS, although a BASFI of $\leq 4(\mathrm{SMD}=2.2,95 \% \mathrm{CI}-0.9$ to $5.3, P=0.16)$ and duration of $>8$ years $(\mathrm{SMD}=11.9$, 95\% CI -10.33 to $34.13, P=0.29$ ) did not (Figs. 3, 4). sRANKL-related factor serum OPG levels $(\mathrm{SMD}=0.86$, 95\% CI 0.09-1.64, $P=0.03$ ) (Fig. 5) in patients with AS were lower than those in controls, and the RANKL/OPG ratio (SMD $=1.05,95 \%$ CI 0.64-1.46, $P<0.00001$ ) (Fig. 5) in patients with AS was higher than that in controls. All results showed that high expression of sRANKL was an important risk factor for the occurrence of AS in the Chinese population.

\section{Sensitivity analysis and publication bias}

The results of the sensitivity analysis indicated that none of the studies had an effect on the overall estimate of the association between RANKL levels and AS risk. Thus, the data presented in our meta-analysis were relatively stable and credible (Fig. 6). The graphical funnel plots of the 12 included studies were symmetrical, and Egger's test showed no publication bias $(P=0.056)$ (Fig. 7).

\section{Discussion}

In this study, we evaluated the sRANKL levels in Chinese patients with AS from 12 articles through meta-analysis, and we investigated the probable relationship between sRANKL level and AS. Our results suggested that RANKL may play a key role in the pathogenesis of AS in Chinese patients. AS is a chronic, progressive systemic rheumatism that affects the sacroiliac joints, central axis bones, peripheral joints, and other extra-articular organs [45]. A recent study suggests that AS pathogenesis involves bone resorption and formation [46]. Although many studies have evaluated the correlation between sRANKL and AS in the Chinese population, their results are controversial [47-49]. The specific number of subjects in the control group of the 47th citation was unclear. The 48th citation does not have RANKL specific data. The

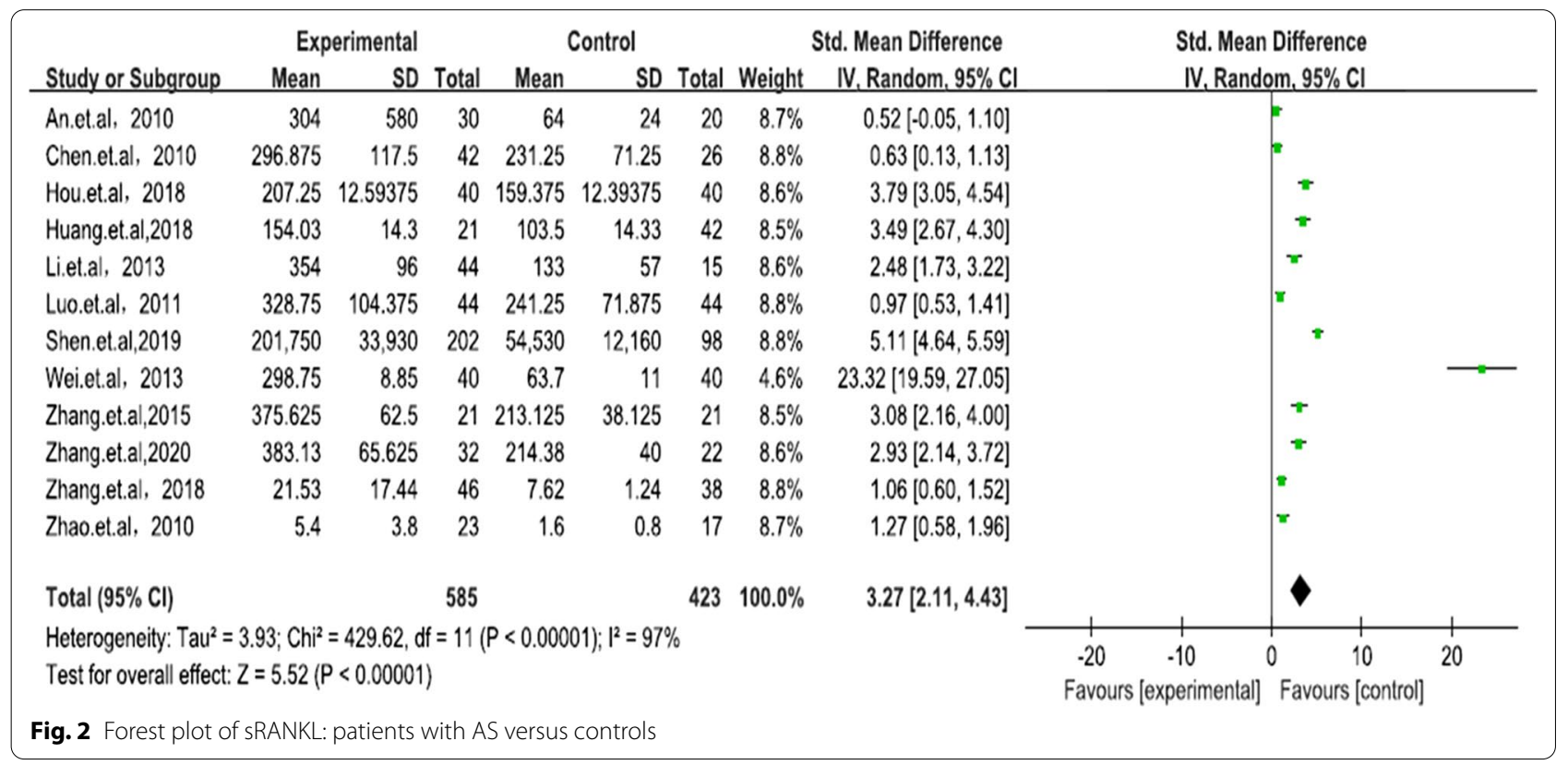




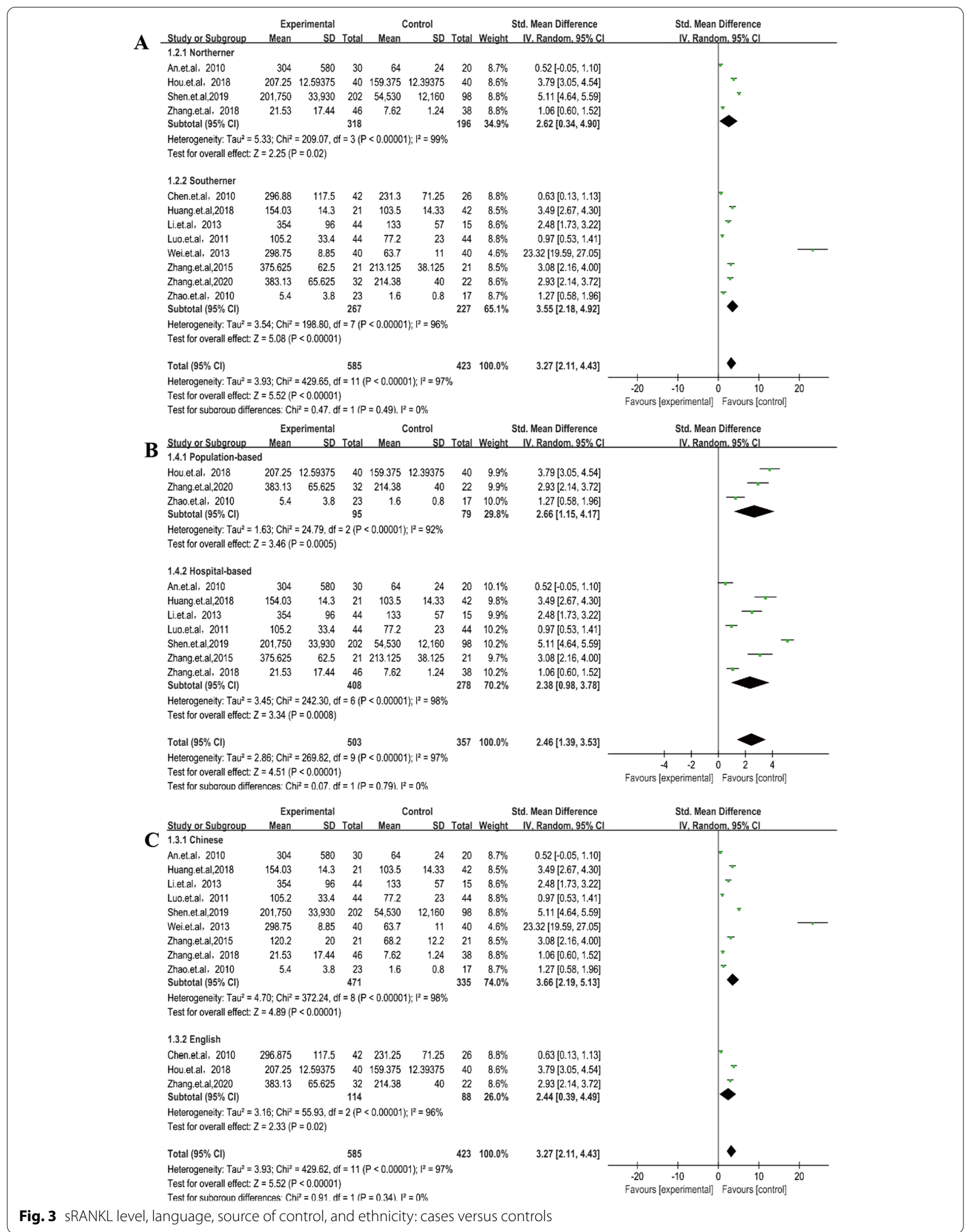




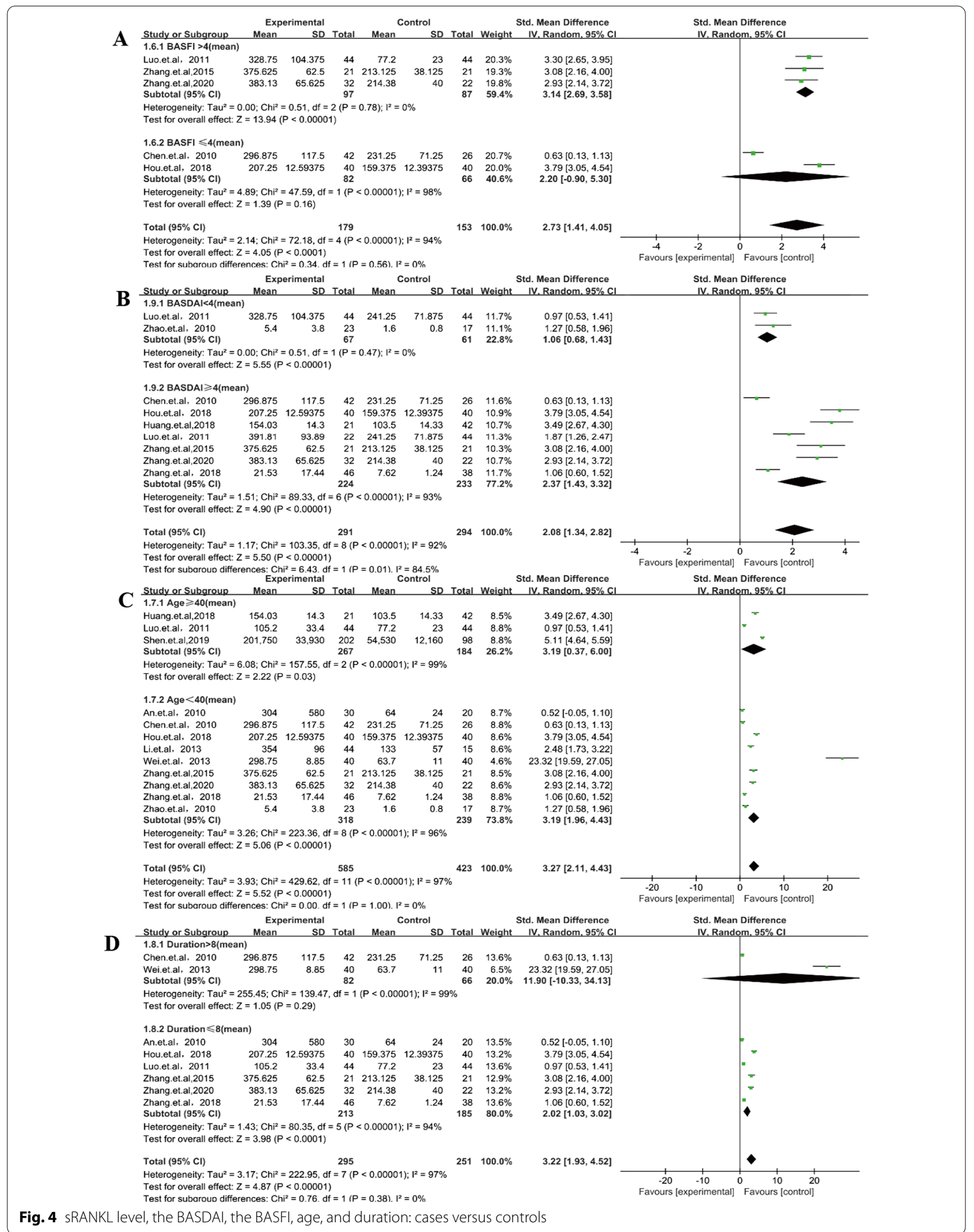




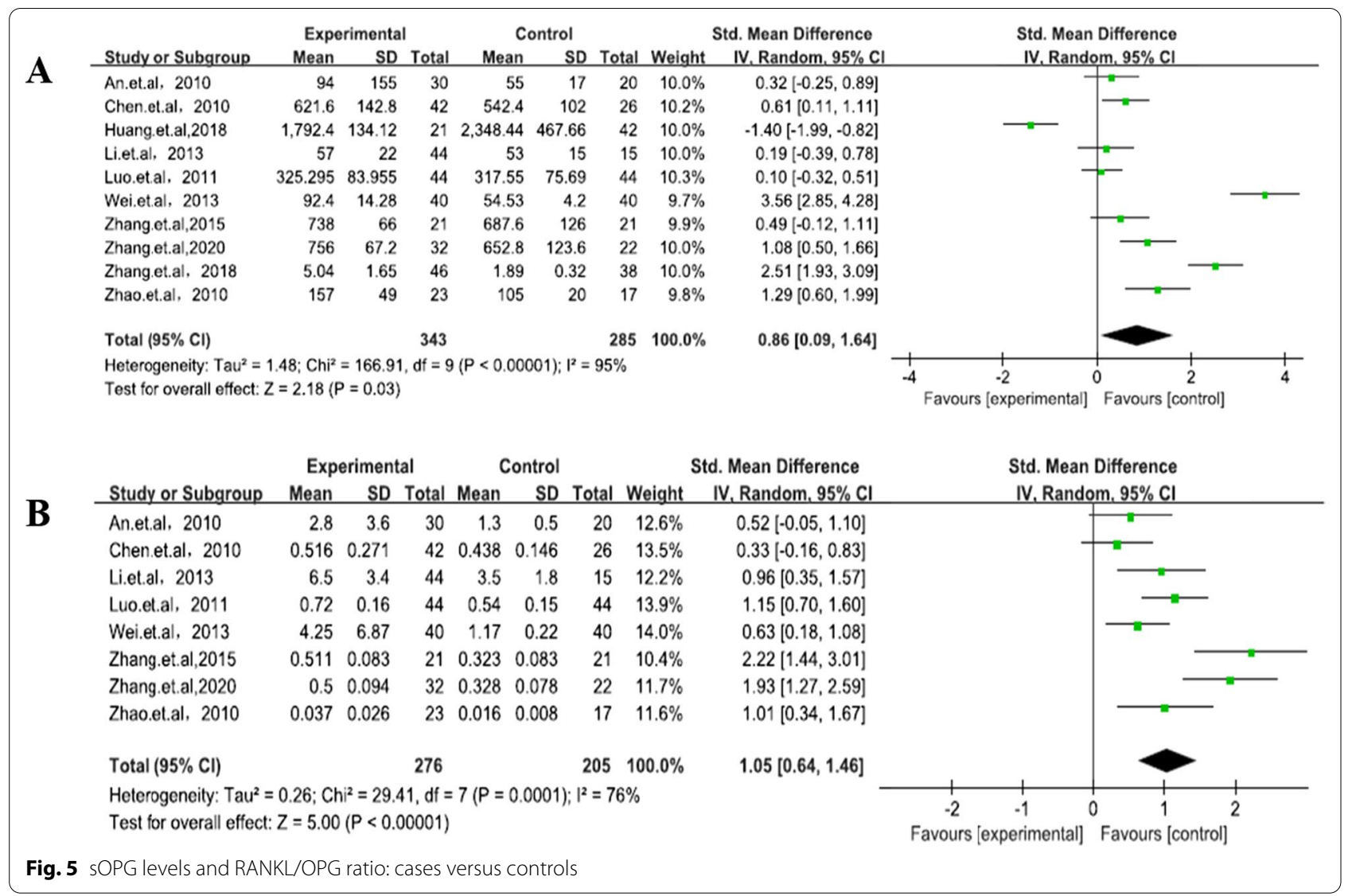

49th citation is a self-control study. Formal requests were made through e-mails to the authors of some studies (the 47th and 48th citations) to obtain their data, although no response was received. Therefore, in line with our screening criteria, these three articles were finally removed. Therefore, we conducted this study to investigate the association between sRANKL and AS. In the process of AS-associated peripheral joint ossification, coupling imbalance between osteoblasts and osteoclasts is a condition that cannot be neglected with regard to ossification [50]. RANKL produced by osteocytes plays an important role in osteoclast formation and bone reconstruction [51]. The RANKL/RANK/OPG pathway controls osteoclastic activity and formation and plays an important role in the pathogenesis of AS [52]. Denosumab is a monoclonal antibody against RANKL that prevents osteoclast formation and has been used as a first-line treatment for osteoporosis. [53] In a study of patients with active Rheumatoid arthritis (RA) 6-12 months of denosumab therapy led to a successful reduction in radiological progress, improved bone mineral density (BMD) of the lumbar spine and entire hip, and significantly reduced bone erosion, along with reduction in bone turnover markers [54]. Another study showed that patients with RA who received $180 \mathrm{mg}$ of denosumab had reduced joint erosion according to MRI measurement at 6 months after treatment [55]. In addition, studies have reported that denosumab treatment can significantly reduce the loss of BMD around the prosthesis after total knee arthroplasty. This treatment strategy can promote early stable fixation of the prosthesis [20]. A study also showed that using tripterygium to reduce the expression of RANKL in the blood can significantly reduce the disease activity in patients with AS [20]. Therefore, lowering the RANKL level in serum may be beneficial to the progression of AS. OPG in bone formation is a kind of protective factor conducive to the growth of osteoblasts, normal osteoblasts, and osteoclasts in the body in a dynamic balance with RANKL; both are not excessively activated but otherwise may lead to bone disease [56]. The RANKL/ OPG ratio is closely related to osteoclast formation and maturity. In this study, the OPG level in peripheral blood of patients with AS (SMD $=0.86$, 95\% CI 0.09-1.64, $P<0.03)$ was significantly lower than of healthy controls, although the sRANKL level $(\mathrm{SMD}=3.27,95 \%$ CI $2.11-$ 4.43, $P<0.00001)$ was significantly higher in patients with AS than in healthy controls. As such, osteoblastic activity was restrained. In-depth studies have shown 


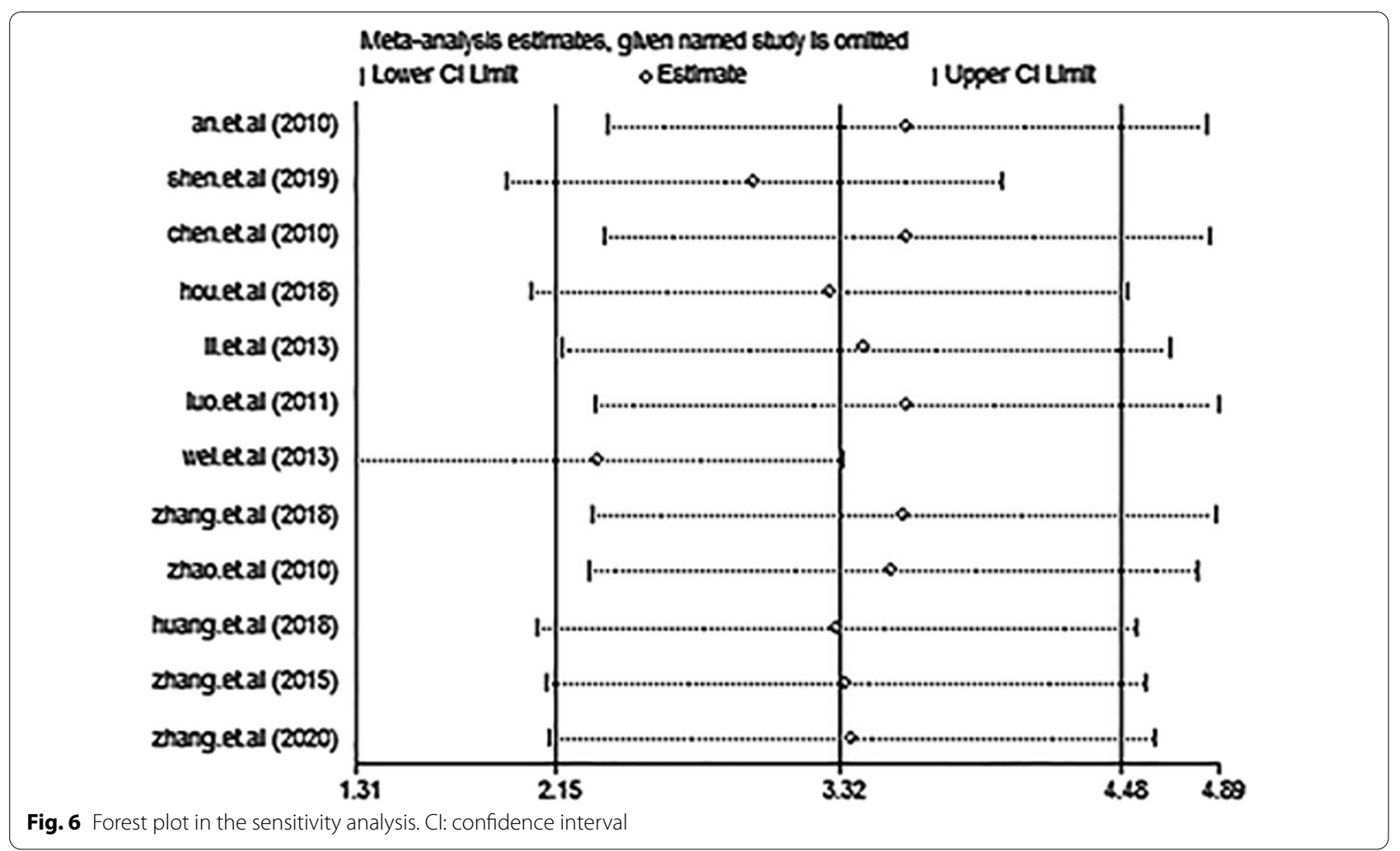

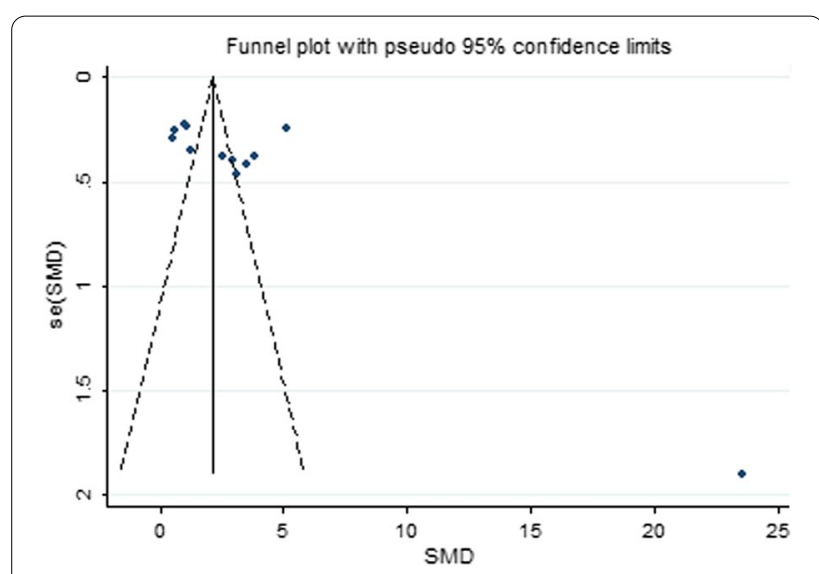

Fig. 7 Shape of the funnel plot. Egger's test, $t=2.16, P=0.056$

that the RANKL/OPG ratio determines the direction of bone change. As the ratio decreases, bone loss decreases [57]. In this study, the RANKL/OPG $(\mathrm{SMD}=1.05$, 95\% CI 0.64-1.46, $P<0.00001)$ ratio was higher in Chinese patients with AS than in controls. This result suggests that excessive activation of osteoclasts increases inflammation as confirmed in animal models. OPG gene knockout mice had insufficient osteoblast production and decreased bone mass, and this leads to severe osteoporosis with a high incidence of bone fracture [58]. The RANKL knockout mice developed severe osteosclerosis, and only a small number of osteoclasts were observed in the bone tissue of those mice $[59,60]$. In addition, RANKL-positive osteocytes were elevated in animal models of inflammation, such as periodontitis and spinal injuries [61]. Osteoblasts and activated $\mathrm{T}$ cells also produce RANKL to regulate adaptive immunity $[12,62]$. Some studies have found that RANKL is expressed on the surface of $T$ cells and lymphocytes, and this regulates lymph node formation and $\mathrm{T}$ cell and dendritic cell communication. Overactivation of the immune system may contribute to the disease process of AS [63]. RANKLexpressed $\mathrm{T}$ cells can affect osteoclast formation, which explains bone loss in patients with chronic inflammatory diseases [16]. Recently, CD4+ T and CD8 $+\mathrm{T}$ cells were confirmed to participate in the pathogenesis of AS, although many problems are yet to be resolved. Furthermore, RANKL overexpression in T cells in RANKL knockout mice can restore osteoclast production and lead to a partial return of the normal bone marrow cavity [64]. Meanwhile, in RANKL deficient mutant mice, the lack of osteoclasts leads to severe osteoporosis and failure of tooth and lymph node formation [65]. Thus, bone loss due to inflammation may arise from the complex interactions of bone cells, $\mathrm{T}$ and $\mathrm{B}$ cells, and signaling 
pathways, such as the RANKL/RANK/OPG pathway [66]. This phenomenon may explain the roles of systemic activation of $\mathrm{T}$ cells and RANKL production through $\mathrm{T}$ cells as important mediators of bone loss in vivo [67]. In autoimmune diseases, arthritis, or local inflammation of the bone caused by infections, $\mathrm{T}$ cells are usually activated first, leading to the overexpression of RANKL and consequent bone loss [68].

Considering that other related factors may have a connection with high levels of sRANKL and AS pathogenesis, a stratified analysis based on region, language, source of control, age, duration, the BASDAI, and the BASFI, was conducted. The BASDAI and BASFI were representative of the activity of the disease [27]. In the current study, sRANKL in the disease group of BASDAI $>4$ and BASFI $>4$ was significantly higher than that in the control group. sRANKL in the subgroup with a disease duration of $>8$ years was significantly higher than that in the control group and may be attributed to the abnormal activation of the immune system and inflammatory cytokine in early AS [69]. The longer the course of the disease, the higher the activity of the disease and the more obvious are the systemic symptoms. It was found in studies with an average course of $>8$ years that the longer the duration of AS, the greater the risk of cardiovascular disease, hearing loss, and poor physical mobility [70, 71]. At the same time, AS causes the lung interstitium to develop lesions at an early stage, and the range of lesions increases with the duration of the disease [72]. In this meta-analysis, we observed similar results. Regardless of age $\geq 40$ years or $<40$ years in the subgroup, the serum RANKL level of patients with AS was significantly higher than that of controls, indicating that age may be an influencing factor on serum RANKL levels in patients with AS. The BASDAI is an indicator for assessing disease activity, and a score of $>4$ indicates that the disease is in the active phase. The BASFI is a recognized standard for assessing the functional ability of patients with AS; the larger the score, the worse the spinal function [73]. Many research reports show that anti-inflammatory treatment can significantly improve the BASDAI and BASFI, and this could also lead to significant reduction in RANKL levels $[74,75]$. Therefore, we speculate that serum RANKL levels may have a potential role in the assessment of inflammation and functional status of patients with AS. All these findings suggest that a BASDAI of $>4$, a BASFI of $>4$, and the duration of the disease affect sRANKL expression. We also found that subgroup $\mathrm{BASDAI}<4$ and BASFI $>4$ are sources of heterogeneity. In terms of region, the relationship was significant among Chinese patients with AS, especially among those patients from the South. This result may be attributed to differences in China's vast territory and geographical distribution of people, genetics, diet, and living environment. Studies have reported that there is a significant delay in diagnosing patients with AS in the south compared with those in the north. This may be due to the large number of mountainous areas in Southern China and inconvenient transportation, which is not conducive for patient examination and treatment, and may lead to the progression of the disease. However, there are many plains in the north and transportation is convenient, which makes it conducive to diagnose and to treat such patients [76]. In fact, HLA-B*2704 is the main subtype of the Chinese population, while HLA-B*2704 is the main subtype of patients with AS, and its carriers are more in South China, compared with those in the north [77]. Some studies have shown that a low starch diet can reduce inflammation and symptoms in patients with AS [78], and a high-fat diet is positively correlated with BASDAI activity. Longterm exposure to PM2.5 is closely related to the BASMI, BASFI, and BASDAI scores of patients with AS. Furthermore Long-term exposure to pollution can cause severe symptoms in patients with AS [79]. Therefore, more indepth research is needed to explore the pathogenesis of AS in Chinese patients in the future.

This meta-analysis has some limitations. First, the small sample size of the 12 studies may have affected our results. Second, articles that provide only medians and ranges or upper quartiles and lower quartiles were excluded because if we converted these data, the result of the transformation may not have been accurate even if a method of transformation had been reported by Hozo et al. [24, 80, 81]. Third, in the 12 studies, information on factors that affect sRANKL, such as HLAB27, body mass index, and sex, were not detailed enough. Therefore, we cannot safely further analyze the relationship between serum RANKL and AS given that the sex ratio between male and female individuals may have an impact on the reliability of our study. Despite the above limitations, this is the first meta-analysis on the association between sRANKL levels in Chinese patients with AS and those in healthy controls.

In conclusion, our study indicated that sRANKL levels in Chinese patients with AS especially in patients with AS in the south, were obviously higher than those in the healthy controls. sRANKL level may have a positive correlation with the pathogenesis of Chinese patients with AS and could serve as a promising biomarker for the severity of AS in Chinese patients. The results of our study may ultimately contribute to the development of new treatments methods for bone damage in Chinese patients with AS, as this field has not been thoroughly studied. Further intensive study on Chinese individuals with a large sample size are needed. 


\section{Conclusions}

The findings of our study suggests that sRANKL has a positive correlation with the pathogenesis of AS in Chinese patients and may potentially serve as a biomarker for the severity of AS in Chinese populations.

\begin{abstract}
Abbreviations
AS: Ankylosing Spondylitis; RANKL: Receptor activator of nuclear factor-kappa B ligand; OPG: Osteoprotegerin; TNF: Tumor Necrosis Factor; BASFI: Bath Ankylosing Spondylitis Functional Index; BASDAl: Bath Ankylosing Spondylitis Disease Activity Index; Cl: Confidence interval; SMD: Standard mean difference; M: Male; F: Female; RA: Rheumatoid arthritis; NOS: Newcastle-Ottawa Scale; HB: Hospital based; PB: Population based; PRISMA: Preferred Reporting Items for Systematic Reviews and Meta-Analyses; ELISA: Enzyme-linked immunosorbent assay.
\end{abstract}

\section{Acknowledgements}

We would like to acknowledge the reviewers for their helpful comments on this paper.

\section{Authors' contributions}

FN designed the study, analyzed most of the data, and wrote manuscript. YZ YP, XP and $J \mathrm{~L}$ proofread the manuscript. All authors read and approved the final manuscript.

\section{Funding}

This work was supported by National Natural Science Foundation of China (81971829).

\section{Availability of data and materials}

The authors confirm that the data supporting the findings of this study are available within the article.

\section{Ethical approval}

As this article is a systematic review and meta-analysis, ethical approval is not needed.

\section{Consent for publication}

Not applicable.

\section{Competing interests}

The authors have no conflicts of interests to declare.

\section{Author details}

'Department of Orthopaedics, Shengjing Hospital of China Medical University, Sanhao Street No. 36, Heping District, Shenyang, Liaoning 110004, People's Republic of China. ${ }^{2}$ Department of Orthopedics, Tianjin Baodi Hospital/Baodi Clinical College of Tianjin Medical University, Tianjin 301800, People's Republic of China. ${ }^{3}$ Department of Urological, Nantong University Danyang Teaching Hospital, Zhenjiang 212300, People's Republic of China. ${ }^{4}$ Daxing Teaching Hospital of Capital Medical University, Beijing 102600, People's Republic of China.

Received: 1 August 2021 Accepted: 13 September 2021

Published online: 18 October 2021

\section{References}

1. Dougados M, Baeten D. Spondyloarthritis. Lancet. 2011;377(9783):2127-37.

2. Zhao $\mathrm{JL}$, et al. Prevalence of ankylosing spondylitis in a Chinese population: a systematic review and meta-analysis. Rheumatol Int. 2020;40(6):859-72.

3. Tu LD, et al. Costs and work limitation of patients with ankylosing spondylitis in China. Clin Exp Rheumatol. 2014;32(5):661-6.

4. Smith JA. Update on ankylosing spondylitis: current concepts in pathogenesis. Curr Allergy Asthma Rep. 2015;15(1):489.
5. Liu W, et al. Elevated serum levels of IL-6 and IL-17 may associate with the development of ankylosing spondylitis. Int J Clin Exp Med. 2015:8(10):17362-76.

6. Wu Y, et al. Risk factors of renal involvement based on different manifestations in patients with ankylosing spondylitis. Kidney Blood Press Res. 2018;43(2):367-77.

7. Maksymowych WP, et al. Serum matrix metalloproteinase 3 is an independent predictor of structural damage progression in patients with ankylosing spondylitis. Arthritis Rheum. 2007;56(6):1846-53.

8. Stupphann D, et al. Intracellular and surface RANKL are differentially regulated in patients with ankylosing spondylitis. Rheumatol Int. 2008;28(10):987-93.

9. Lacey $\mathrm{DL}$, et al. Osteoprotegerin ligand is a cytokine that regulates osteoclast differentiation and activation. Cell. 1998:93(2):165-76.

10. Yasuda $\mathrm{H}$, et al. Identity of osteoclastogenesis inhibitory factor (OCIF) and osteoprotegerin (OPG): a mechanism by which OPG/OCIF inhibits osteoclastogenesis in vitro. Endocrinology. 1998;139(3):1329-37.

11. Ma Q, et al. Mature osteoclast-derived apoptotic bodies promote osteogenic differentiation via RANKL-mediated reverse signaling. J Biol Chem. 2019:294(29):11240-7.

12. Anderson DM, et al. A homologue of the TNF receptor and its ligand enhance T-cell growth and dendritic-cell function. Nature. 1997;390(6656):175-9.

13. Amin N, et al. Probiotics and bone disorders: the role of RANKL/RANK OPG pathway. Aging Clin Exp Res. 2020;32(3):363-71.

14. Nagy $V$, Penninger JM. The RANKL-RANK Story. Gerontology. 2015:61(6):534-42

15. Hofbauer LC, Heufelder AE. Role of receptor activator of nuclear factorkappaB ligand and osteoprotegerin in bone cell biology. J Mol Med. 2001;79(5-6):243-53.

16. Kong $Y Y$, et al. OPGL is a key regulator of osteoclastogenesis, lymphocyte development and lymph-node organogenesis. Nature. 1999:397(6717):315-23.

17. Nakashima TM, Hayashi TH. New insights into osteoclastogenic signaling mechanisms. Trends Endocrinol Metab. 2012;23(11):582-90.

18. Jones RM, Mulle JG, Pacifici R. Osteomicrobiology: The influence of gut microbiota on bone in health and disease. Bone. 2018;115:59-67.

19. de Vries TJ, Huesa C. The osteocyte as a novel key player in understanding periodontitis through its expression of RANKL and sclerostin: a review. Curr Osteoporos Rep. 2019:17(3):116-21.

20. Ji W, et al. Beneficial effects of tripterygium glycosides tablet on biomarkers in patients with ankylosing spondylitis. Mol Med Rep. 2015;12(1):684-90.

21. Mou YK, et al. Changes of serum levels of MMP-3, sRANKL, and OPG in juvenile-onset ankylosing spondylitis patients carrying different HLA-B27 subtypes. Clin Rheumatol. 2015;34(6):1085-9.

22. Du W, et al. MiR-495 targeting dvl-2 represses the inflammatory response of ankylosing spondylitis. Am J Transl Res. 2019;11(5):2742-53.

23. Dhir VR, Srivastava AA. Circulating levels of soluble receptor activator of NF-K B ligand and matrix metalloproteinase 3 (and their antagonists) in Asian Indian patients with ankylosing spondylitis. Int J Rheumatol. 2013;2013:814350.

24. Taylan A, et al. Biomarkers and cytokines of bone turnover: extensive evaluation in a cohort of patients with ankylosing spondylitis. BMC Musculoskelet Disord. 2012;13:191.

25. Van der Linden S, Valkenburg HA, Cats A. Evaluation of diagnostic criteria for ankylosing spondylitis. A proposal for modification of the New York criteria. Arthritis Rheum. 1984;27(4):361-8.

26. Sieper J, et al. The Assessment of SpondyloArthritis international Society (ASAS) handbook: a guide to assess spondyloarthritis. Ann Rheum Dis. 2009;68(Suppl 2):ii 1-44.

27. Zochling J. Measures of symptoms and disease status in ankylosing spon dylitis: Ankylosing Spondylitis Disease Activity Score (ASDAS), Ankylosing Spondylitis Quality of Life Scale (ASQoL), Bath Ankylosing Spondylitis Disease Activity Index (BASDAI), Bath Ankylosing Spondylitis Functional Index (BASFI), Bath Ankylosing Spondylitis Global Score (BAS-G), Bath Ankylosing Spondylitis Metrology Index (BASMI), Dougados Functional Index (DFI), and Health Assessment Questionnaire for the Spondylarthropathies (HAQ-S). Arthritis Care Res (Hoboken). 2011;63(Supp 11):S47-58 
28. Stang A. Critical evaluation of the Newcastle-Ottawa scale for the assessment of the quality of nonrandomized studies in meta-analyses. Eur J Epidemiol. 2010;25(9):603-5.

29. Zintzaras E, loannidis JP. Heterogeneity testing in meta-analysis of genome searches. Genet Epidemiol. 2005;28(2):123-37.

30. Higgins JP, Thompson SG. Quantifying heterogeneity in a meta-analysis. Stat Med. 2002;21(11):1539-58.

31. Song F, Gilbody S. Bias in meta-analysis detected by a simple, graphical test. Increase in studies of publication bias coincided with increasing use of meta-analysis. BMJ. 1998;316(7129):471.

32. Peters JL, et al. Comparison of two methods to detect publication bias in meta-analysis. JAMA. 2006;295(6):676-80.

33. An XB, et al. Analysis of related factor osteoporosis in ankylosing spondylitis. Chin J Rheumatol. 2010;14(9):620-3.

34. Chen $\mathrm{CH}$, et al. Soluble receptor activator of nuclear factor-kappaB ligand (RANKL) and osteoprotegerin in ankylosing spondylitis: OPG is associated with poor physical mobility and reflects systemic inflammation. Clin Rheumatol. 2010;29(10):1155-61.

35. Hou CL, Luan L, Ren CF. Oxidized low-density lipoprotein promotes osteoclast differentiation from CD68 positive mononuclear cells by regulating HMGB1 release. Biochem Biophys Res Commun. 2018;495(1):1356-62.

36. Li XJ, et al. The study on the expression of interieukin 17 and receptor activator of nuclear factors KB-ligand in serum of patients with Ankylosing spondylitis. Chin J Rheumatol. 2013;17(11):769-71.

37. Luo HY. Expression and signifinance of serum OPG and SRANKL in patients with Ankylosing spondylitis. Jiangsu Med. 2011;37(23):2770-2.

38. Wei RM, et al. Correlation of bone metabolic indexes and levels of rankl-rank-opg system in ankylosing spondylitis patients. Progress Mod Biomed. 2013;13(13): 2528-2531, 2546

39. Zhang Z, et al. Clinical significance of detection of MIF, IL23, RANKL, OPG and DKK1 in peripheral blood of patients with active ankylosing spondylitis. Hebei Med J. 2018;40(02):178-82.

40. Zhao WH, et al. Osteoclast precursors in perip heral blood of patients with Ankylosing spondylitis. Chin J Rheumatol. 2010;14(6):373-6.

41. Shen J, et al. Clinical significance of RANKL, TGF- $\beta 1$ and TGF- $\beta 2$ in ankylosing spondylitis. Shaanxi Med J. 2019;48(08):981-3.

42. Huang $X Q$, et al. Correlation of serum TNF - a, RANKL, OPG and IL-34 levels with enthesitis in patients with ankylosing spondylitis. Zhejiang Med J. 2018;40(22):2454-8.

43. Zhang PY, et al. Expression of chemokine CXCL16 and its receptor CXCR6 can be suppressed by rcombinant human TNF receptora IIIg fusion protein in ankylosing spondylitis. J Shandong Univ (Health Sci). 2015;53(12):5156

44. Zhang P, et al. TNF receptor: Fc fusion protein downregulates RANKL/OPG ratio by inhibiting CXCL16/CXCR6 in active ankylosing spondylitis. Curr Pharm Biotechnol. 2021;22(2):305-16.

45. Gouveia EB, Elmann D, Morales MS. Ankylosing spondylitis and uveitis: overview. Rev Bras Reumatol. 2012;52(5):742-56.

46. Cortes A, et al. Association study of genes related to bone formation and resorption and the extent of radiographic change in ankylosing spondylitis. Ann Rheum Dis. 2015;74(7):1387-93.

47. Kim HR, et al. Elevated serum levels of soluble receptor activator of nuclear factors-kappaB ligand (sRANKL) and reduced bone mineral density in patients with ankylosing spondylitis (AS). Rheumatology (Oxford). 2006:45(10):1197-200.

48. Fan J, et al. I-BET151 inhibits expression of RANKL, OPG, MMP3 and MMP9 in ankylosing spondylitis and. Exp Ther Med. 2017;14(5):4602-6.

49. Holmdahl $\mathrm{R}$, et al. Collagen induced arthritis as an experimental model for rheumatoid arthritis. Immunogenetics, pathogenesis and autoimmunity. APMIS. 1989;97(7):575-84

50. Nakashima T, et al. Evidence for osteocyte regulation of bone homeostasis through RANKL expression. Nat Med. 2011;17(10):1231-4.

51. Jones DH, Kong YY, Penninger JM. Role of RANKL and RANK in bone loss and arthritis. Ann Rheum Dis. 2002;61 Suppl 2:ii32-9.

52. Hinze AM, Louie GH. Osteoporosis management in ankylosing spondylitis. Curr Treatm Opt Rheumatol. 2016;2(4):271-82.

53. Dubrovsky AM, Lim MJ, Lane NE. Osteoporosis in rheumatic diseases: anti-rheumatic drugs and the skeleton. Calcif Tissue Int 2018;102(5):607-18.

54. Cohen SB, et al. Denosumab treatment effects on structural damage, bone mineral density, and bone turnover in rheumatoid arthritis: a twelve-month, multicenter, randomized, double-blind, placebo-controlled, phase II clinical trial. Arthritis Rheum. 2008;58(5):1299-309.

55. Murahashi Y, et al. Denosumab prevents periprosthetic bone mineral density loss in the tibial metaphysis in total knee arthroplasty. Knee. 2020;27(2):580-6.

56. Kovács B, Vajda E, Nagy EE. Regulatory effects and interactions of the Wnt and OPG-RANKL-RANK Signaling at the Bone-Cartilage Interface in Osteoarthritis. Int J Mol Sci. 2019;20(18):4653.

57. Tobeiha M, et al. RANKL/RANK/OPG pathway: a mechanism involved in exercise-induced bone remodeling. Biomed Res Int. 2020;2020:6910312.

58. Ozaki Y, et al. Treatment of OPG-deficient mice with WP9QY, a RANKL-binding peptide, recovers alveolar bone loss by suppressing osteoclastogenesis and enhancing osteoblastogenesis. PLOS ONE. 2017;12(9):e0184904.

59. Hu Z, et al. Serum from patients with ankylosing spondylitis can increase PPARD, fra-1, MMP7, OPG and RANKL expression in MG63 cells. Clinics (Sao Paulo). 2015;70(11):738-42

60. Atkinson SM, et al. Anti-RANKL treatment inhibits erosive joint destruction and lowers inflammation but has no effect on bone formation in the delayed-type hypersensitivity arthritis (DTHA) model. Arthritis Res Ther. 2016;18:28.

61. Metzger CE, Narayanan SA. The role of osteocytes in inflammatory bone loss. Front Endocrinol (Lausanne). 2019;10:285.

62. Wong BR, et al. TRANCE is a novel ligand of the tumor necrosis factor receptor family that activates c-Jun $\mathrm{N}$-terminal kinase in T cells. J Biol Chem. 1997;272(40):25190-4.

63. Varsani $H$, et al. Synovial dendritic cells in juvenile idiopathic arthritis (JIA) express receptor activator of NF-kappaB (RANK). Rheumatology (Oxford). 2003;42(4):583-90.

64. Kim N, et al. Diverse roles of the tumor necrosis factor family member TRANCE in skeletal physiology revealed by TRANCE deficiency and partial rescue by a lymphocyte-expressed TRANCE transgene. Proc Natl Acad Sci USA. 2000;97(20):10905-10.

65. Dankbar B, et al. Myostatin is a direct regulator of osteoclast differentiation and its inhibition reduces inflammatory joint destruction in mice. Nat Med. 2015;21(9):1085-90.

66. Di Munno O, Ferro F. The effect of biologic agents on bone homeostasis in chronic inflammatory rheumatic diseases. Clin Exp Rheumatol. 2019;37(3):502-7.

67. Hoffmann DB, et al. In vivo siRNA delivery using JC virus-like particles decreases the expression of RANKL in rats. Mol Ther Nucleic Acids. 2016;5:e298.

68. Wei CM, et al. Monocrotaline suppresses RANKL-induced osteoclastogenesis in vitro and prevents LPS-induced bone loss in vivo. Cell Physiol Biochem. 2018;48(2):644-56.

69. Watad A, et al. The early phases of ankylosing spondylitis: emerging insights from clinical and basic science. Front Immunol. 2018;9:2668.

70. Sarp U, et al. Biomarkers of cardiovascular disease in patients with ankylosing spondylitis. Arch Rheumatol. 2020;35(3):435-9.

71. Ajmani S, et al. Hearing loss in ankylosing spondylitis. Int J Rheum Dis. 2019;22(7):1202-8

72. Senocak $\mathrm{O}$, et al. Lung parenchyma changes in ankylosing spondylitis: demonstration with high resolution CT and correlation with disease duration. Eur J Radiol. 2003;45(2):117-22.

73. Abdal SJ, et al. Development of a Bangla version of the Bath Ankylosing Spondylitis Disease Activity Index (BASDAl) and the Bath Ankylosing Spondylitis Functional Index (BASFI). Int J Rheum Dis. 2021;24(1):74-80.

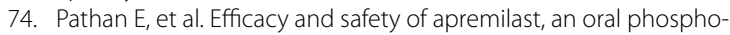
diesterase 4 inhibitor, in ankylosing spondylitis. Ann Rheum Dis. 2013;72(9):1475-80.

75. Tanski W, et al. Benefit of biological drugs for quality of life in patients with ankylosing spondylitis: a systematic review and meta-analysis of clinical trials. Adv Exp Med Biol. 2020;25:320.

76. Ma HJ, et al. Different clinical features in patients with ankylosing spondylitis from southern and northern China. Int J Rheum Dis. 2012;15(2):154-62.

77. Luo F, et al. Comparison of HLA-B*27 subtypes between Chinese patients with ankylosing spondylitis and non-ankylosing spondylitis carriers. J Int Med Res. 2019:47(7):3171-8. 
78. Rashid T, Wilson C, Ebringer A. Raised incidence of ankylosing spondylitis among Inuit populations could be due to high HLA-B27 association and starch consumption. Rheumatol Int. 2015;35(6):945-51.

79. Soleimanifar $\mathrm{N}$, et al. Effect of food intake and ambient air pollution exposure on ankylosing spondylitis disease activity. Adv Rheumatol. 2019;59(1):9.

80. Klingberg $\mathrm{E}$, et al. Biomarkers of bone metabolism in ankylosing spondylitis in relation to osteoproliferation and osteoporosis. J Rheumatol. 2014:41(7):1349-56.
81. Wan $\mathrm{X}$, et al. Estimating the sample mean and standard deviation from the sample size, median, range and/or interquartile range. BMC Med Res Methodol. 2014;14:135.

\section{Publisher's Note}

Springer Nature remains neutral with regard to jurisdictional claims in published maps and institutional affiliations.
Ready to submit your research? Choose BMC and benefit from:

- fast, convenient online submission

- thorough peer review by experienced researchers in your field

- rapid publication on acceptance

- support for research data, including large and complex data types

- gold Open Access which fosters wider collaboration and increased citations

- maximum visibility for your research: over $100 \mathrm{M}$ website views per year

At BMC, research is always in progress.

Learn more biomedcentral.com/submissions 